\title{
PROGNOSTIC INDICATORS IN IDIOPATHIC SUDDEN SENSORINEURAL HEARING LOSS IN A MALAYSIAN HOSPITAL
}

\author{
Dr Tiong S, UNIMAS, Kuching, Malaysia
}

Objectives: To review the prognostic indicators in management of idiopathic sudden sensorimeural hearing loss in otorhinolaryngology patients treated in Sarawak General Hospital., Malaysia.

Methods: This review covered the 3 years 2001 to 2003 on all patients treated for sensorineural hearing loss in outpatient clinic of the Hospital. 8 patients with known causes of hearing loss were excluded and the remaining 66 patients were studied. All patients were followed up progressively 3 to 12 months and pure tone audiograms done on initial and follow-up visits. The audiograms on hearing threshold increments of $5 \mathrm{~dB}$ and the frequencies in $\mathrm{KHz} 0.25$ to 8.0 were done. The hearing improvement is taken as improvement from initial thresholds by more than $20 \mathrm{~dB}$ in at least 2 contiguous frequencies. Their hearing improvement in relation to pure tone audiogram patterns, hearing loss severity, vertigo, timnitus, age of the patients and durations from symptom onset to time of treatment, were evaluated.

Results: There were 34 females and 32 males, with age from 15 to 80 years and mean age of 49 (SD 16.91). 64 patients had unilateral SSNHL and 2 bilateral. Hearing improvement was noted in 42 patients $(64 \%)$. Six audiogram patterns were observed with good prognostic indicators in patterns 1 (sloping -up, $91 \%$ )and 4 ( mid-dipping, 100\%). The hearing improvement was noted in majority of the patients with mild or moderate degree of hearing loss. Patients aged 21 to 40 appear to have better prognosis than those aged younger or older. Treatment started in the first or second week since onset of hearing loss had better hearing improvement $(79 \%, 76 \%)$ than started in later weeks $(<50 \%)$. Percentages of hearing improvement when tinnitus absent or present are much the same. Poor prognosis is noted in vertigo $(40 \%$ or less) than without vertigo $(74 \%)$.

Conclusion: Good prognostic indicators in this study are age 21 to 40 , mild or moderate hearing loss, no vertigo, treatment started first 2 weeks from onset of hearing loss, sloping-up and mid-dipping audiograms.

Contact person email: stiong2005@hotmail.com 